\title{
Optical Resolution and Racemization Mechanism of a Tellurinic Acid
}

Yusuke Nakashima, Toshio Shimizu,* Kazunori Hirabayashi, and Nobumasa Kamigata* Department of Chemistry, Graduate School of Science, Tokyo Metropolitan University Minami-ohsawa, Hachioji, Tokyo 192-0397, Japan

General. Dichloromethane, hexane, and 2-propanol were distilled from $\mathrm{CaH}_{2}$ before use. Melting points were determined on a Yamato MP-21 melting point apparatus. UV-VIS spectra were measured on a UV-3100PC UV-VIS-NIR scanning spectrometer. IR spectra were measured on a PERKIN ELMER Spectrum GX FT-IR system. ${ }^{1} \mathrm{H}$ NMR spectra were recorded on a JEOL JNM-EX-500 FT NMR System. Mass spectra (MS) were determined on JEOL JMS-LX1000 and JEOL JMS-GCMATE System. Circular dichroism spectra were measured on JASCO J-725 Spectropolarimeter. Specific rotations were measured on JASCO DIP-140 Digital polarimeter. Optical rotations are given in $10^{-1} \mathrm{deg}_{\mathrm{cm}} \mathrm{g}^{-1}$.

\section{2,4,6-Triisopropylbenzenetellurinic Acid (1). Bis(2,4,6-triisopropylphenyl)} ditelluride (2.98 g, $4.50 \mathrm{mmol})$ was dissolved in dichloromethane (100 ml) and ozone was bubbled into the solution at $-40{ }^{\circ} \mathrm{C}$. After disappearance of color for the ditelluride, water $(100 \mathrm{ml})$ was added to the solution, and the mixture was stirred vigorously for $2 \mathrm{~h}$ at room temperature. The organic layer was separated, and the organic component remaining in the aqueous layer was extracted with dichloromethane $(25 \mathrm{ml} \mathrm{x} \mathrm{4})$, and the combined organic layer was dried over anhydrous magnesium sulfate. The solution was concentrated to small volume (ca. $5 \mathrm{ml})$ under reduced pressure. Methanol $(50 \mathrm{ml})$ was added to the solution and cooled to $-17{ }^{\circ} \mathrm{C}$. Filtering the white precipitate and drying in vacuo afforded tellurinic acid (1.06 g, 32\%): mp $209{ }^{\circ} \mathrm{C}$ (colorless powder from methanol; decomp.); ${ }^{1} \mathrm{H}$ NMR $\left(500 \mathrm{MHz}, \mathrm{CDCl}_{3}\right) \delta-0.3-2.0(18 \mathrm{H}, \mathrm{br}), 2.81(3 \mathrm{H}$, br), 4.14 (1H, br), 7.03 (2H, br); MS (EI, $30 \mathrm{eV})$ m/z 332, 330, 203, 189, 91; MS (FAB)

m/z $365\left({ }^{130} \mathrm{Te}, \mathrm{M}^{+}-\mathrm{H}\right), 363\left({ }^{128} \mathrm{Te}, \mathrm{M}^{+}-\mathrm{H}\right)$; IR (KBr) 3393 (br, OH), 2960, 2867, 2360, 
1595, 1463, 1103, 878, 647 (br, Te=O) $\mathrm{cm}^{-1}$; UV (hexane) $\lambda_{\max } 279\left(\mathrm{sh}, \varepsilon 3.30 \times 10^{3}\right)$, $249\left(\mathrm{sh}, \varepsilon 1.08 \times 10^{4}\right), 197\left(\varepsilon 6.70 \times 10^{4}\right) \mathrm{nm}$.

(+)-1. Mp $208{ }^{\circ} \mathrm{C}$ (colorless powder; decomp.); $[\alpha]_{435}^{28} 2.5 \times 10^{3}$ (c 0.0012, hexane); $\mathrm{CD}$ (hexane) $270\left([\theta]+1.27 \times 10^{4}\right), 238\left([\theta]-2.18 \times 10^{4}\right) \mathrm{nm}$. Optical purity could not be determined by HPLC nor ${ }^{1} \mathrm{H}$ NMR using Eu(hfc) $)_{3}$.

Theoretical Study. Geometries were optimized using the MP2 method with the LANL2DZ basis set. All calculations were performed by using the Gaussian98 program on an IBM p690-681 (RegattaH) computer. Vibrational frequency analysis of each geometry of transition states of vertex inversion and edge inversion of chalcogenic acids showed one imaginary frequency which corresponds to the vertex inversion or edge inversion mode, clearly indicating the real saddle-point in the reaction pathway. Differences in the zero-points energies between the saddle-points and the ground states are within $0.56 \mathrm{kcal} \mathrm{mol}^{-1}$ in each tellurinic acid, and thus the energies are uncorrected.

\section{Benzentellurinic acid:}

ground state: Energy $=-388.91893730754$ hartree

Cartesian coordinates: Charge $=0$, Multiplicity $=1$

$\begin{array}{llll}\mathrm{Te} & 1.315647 & -.041487 & -.275661 \\ \mathrm{O} & 1.596102 & 1.669684 & .353441 \\ \mathrm{C} & -.830056 & -.031247 & -.137046 \\ \mathrm{C} & -1.466392 & 1.212159 & .154856 \\ \mathrm{C} & -2.885662 & 1.255079 & .278984 \\ \mathrm{C} & -3.658112 & .069612 & .106548 \\ \mathrm{C} & -3.014585 & -1.169670 & -.183994 \\ \mathrm{C} & -1.595954 & -1.224117 & -.310120 \\ \mathrm{O} & 1.509717 & -1.357096 & 1.218792 \\ \mathrm{H} & -3.608136 & -2.080667 & -.310021 \\ \mathrm{H} & -1.104402 & -2.182807 & -.512032\end{array}$




$\begin{array}{rrrr}\mathrm{H} & -.855006 & 2.110104 & .299547 \\ \mathrm{H} & -3.382094 & 2.203440 & .507381 \\ \mathrm{H} & -4.747937 & .109976 & .200355 \\ \mathrm{H} & 1.141926 & -1.174333 & 2.115910\end{array}$

Transition state for verdex inversion: Energy $=-388.78861297387$ hartree Imaginary frequencies $=1$

Cartesian coordinates: Charge $=0$, Multiplicity $=1$

$\begin{array}{lrrr}\mathrm{Te} & 1.205376 & -.038167 & -.069838 \\ \mathrm{O} & 2.561651 & -.779459 & -1.178736 \\ \mathrm{O} & 1.758370 & 1.072150 & 1.511421 \\ \mathrm{C} & -.956003 & -.058852 & -.032993 \\ \mathrm{C} & -1.615892 & 1.084880 & -.574304 \\ \mathrm{C} & -3.041554 & 1.111511 & -.556273 \\ \mathrm{C} & -3.780287 & .013920 & -.027703 \\ \mathrm{C} & -3.099486 & -1.123204 & .495422 \\ \mathrm{C} & -1.674090 & -1.170304 & .502440 \\ \mathrm{H} & 2.059381 & .557288 & 2.301237 \\ \mathrm{H} & -3.563545 & 1.982393 & -.963487 \\ \mathrm{H} & -1.044525 & 1.916325 & -.989916 \\ \mathrm{H} & -1.147902 & -2.035724 & .910345 \\ \mathrm{H} & -3.665536 & -1.968357 & .898253 \\ \mathrm{H} & -4.873720 & .043511 & -.025893\end{array}$

Transition state for edge inversion: Energy $=-388.87790175968$ hartree Imaginary frequencies $=1$

Cartesian coordinates: Charge $=0$, Multiplicity $=1$ 


$\begin{array}{lrrr}\mathrm{Te} & .000014291 & -.000000369 & -.000001735 \\ \mathrm{O} & -.000005255 & .000002066 & -.000004090 \\ \mathrm{C} & .000000390 & .000007614 & .000015279 \\ \mathrm{O} & -.000008578 & -.000002868 & .000005422 \\ \mathrm{C} & -.000003299 & -.000002118 & .000000684 \\ \mathrm{C} & -.000001267 & -.000000405 & -.000003722 \\ \mathrm{C} & .000002610 & .000002120 & -.000006368 \\ \mathrm{C} & -.000000042 & .000000052 & .000019681 \\ \mathrm{C} & -.000001597 & -.000006180 & -.000008955 \\ \mathrm{H} & .000002432 & -.000001827 & -.000005617 \\ \mathrm{H} & -.000001262 & -.000000386 & .000003238 \\ \mathrm{H} & .000000975 & .000000314 & -.000002035 \\ \mathrm{H} & .000001928 & .000001571 & -.000002026 \\ \mathrm{H} & -.000001002 & .000000731 & -.000006673 \\ \mathrm{H} & -.000000324 & -.000000317 & -.000003084\end{array}$

